\title{
Asymptomatic EBV Infection may Uncover Ezetimibe Liver Alterations in a Hypercholesterolemic Child
}

\author{
Giuliana Mombellia, c, Laura Calabresi ${ }^{\mathrm{b}}$, Cesare R Sirtori ${ }^{\mathrm{a}, \mathrm{b}}$
}

\begin{abstract}
The Epstein Barr viral (EBV) infection may be associated with liver side-effects from ezetimibe treatment in children. An 11-year-old child with familial hypercholesterolemia $(\mathrm{FH})$ presented with a normal biochemical liver profile before ezetimibe treatment, with no significant alterations of liver enzymes. Three months after beginning treatment with ezetimibe $10 \mathrm{mg} / \mathrm{day}$, he complained of constant weakness, and blood testing revealed marked transaminase elevations. Administration of ezetimibe was stopped. However 15 days later alanine transferase (ALT) levels showed a further increase, followed, 10 days later, by an improvement in all pathological levels. After a further 2 months, all values had returned to normal. Tests for antibodies to the EBV were positive, suggesting a recent but not acute EBV infection. It was thus decided to test ezetimibe $10 \mathrm{mg}$ /day again, and after 1 month aspartate aminotransferase (AST) and ALT levels were in a normal range, together with a very satisfactory LDL-cholesterol (LDL-C) response. This is the first report of a child with significant liver side-effects from ezetimibe treatment. Ezetimibe treatment may not have been the direct cause of liver damage in this child, because a concomitant EBV infection. Association of the two should be a warning for possible liver complications.
\end{abstract}

Keywords: Liver alterations; Ezetimibe; Epstein Barr virus

\section{Introduction}

Drug treatment of hypercholesterolemia has recently been extended to children with familial disease, following vast

\footnotetext{
Manuscript accepted for publication September 24, 2012

${ }^{a}$ University Center for Dyslipidemias, Niguarda Hospital, piazza Ospedale Maggiore 3, 20162 Milan, Italy

${ }^{b}$ Department of Pharmacological Sciences, University of Milano via Balzaretti 9, 20133 Milano, Italy

${ }^{\mathrm{c} C}$ Corresponding author: Giuliana Mombelli, University Center for Dyslipidemias, Niguarda Hospital, piazza Ospedale Maggiore 3,20162 Milan, Italy. Email: giuliana.mombelli@ospedaleniguarda.it
}

doi: http://dx.doi.org/10.4021/jmc903w clinical trials indicating that pharmacological reduction of hypercholesterolemia (generally by statins) can reduce the progression of the carotid intima media thickness (IMT) in children starting treatment around 8 years of age [1]. Recommendations for drug therapy in children with significant elevations of LDL-C have been endorsed by the National Cholesterol Education Program Pediatric Guidelines [2].

In view of the not rare occurrence of side effects to statins, in particular of the muscular type (most frequently myopathy, in rare cases rhabdomyolysis) [3] some physicians are reluctant to use statins in children. Furthermore, in childhood, the use of a cholesterol lowering agent affecting biosynthesis may, in general, carry some reservations based on animal findings [4], although not strongly supported in the clinic. Thus, it has become of growing interest the use of a drug mainly affecting intestinal lipid absorption, such as ezetimibe. Ezetimibe appears to act mainly on the NPLC-1 system on the intestinal brush border [5], thus reducing the interaction with intestinal sterols. Many authors, however, believe that the drug predominantly affects lipid packing, in this way reducing sterol absorption [6].Data available from children, both carriers of FH and also of polygenic hypercholesterolemia, in general agree on the safety and good tolerability of the agent [7]. The clinical overview of the safety of ezetimibe concluded for adequate acceptability [8]. Furthermore, in a study in children from 10 to 18 years of age, metabolism and pharmacokinetics of ezetimibe were similar to those reported in adults [9]. Only a small number of reports indicate definite liver damage in adults. Stolk et al reported that the drug may rarely cause severe idiosyncratic cholestatic hepatitis or autoimmune hepatitis [10]. We report herein the case of a child treated for severe $\mathrm{FH}$ with ezetimibe $10 \mathrm{mg} /$ daily, presenting markedly increased transaminase levels.

\section{Case Report}

An 11-year-old child with FH was diagnosed as a carrier of the disease at the age of 4 . His family history was quite dramatic, his grandfather dying of a heart attack at age 35 ; his father has a severe form of FH, fairly well controlled with statins. The lipid profile of the child before drug treatment 
was: total cholesterol (TC) $381 \mathrm{mg} / \mathrm{dL}$, LDL-C $313 \mathrm{mg} / \mathrm{dL}$, HDL-cholesterol $60 \mathrm{mg} / \mathrm{dL}$ and triglycerides (TG) $40 \mathrm{mg} /$ dL. His liver biochemical profile was normal with no significant alterations of liver enzyme or bilirubin levels.

He attended our Lipid Clinic for several years, and was prescribed drug treatment in April 2011. His family refused statin treatment and he was thus started on ezetimibe $10 \mathrm{mg} /$ day. In July 2011 he complained of constant weakness, and toxicity testing was carried out. Liver function levels showed marked transaminase elevations, namely AST $65 \mathrm{U} / \mathrm{L}$, ALT $95 \mathrm{U} / \mathrm{L}$, gamma-glutamyl transferase (GGT) $16 \mathrm{U} / \mathrm{L}$ and creatine kinase (CK) $248 \mathrm{U} / \mathrm{L}$; LDL-C levels had decreased to $198 \mathrm{mg} / \mathrm{dL}$. At this point, it was decided to stop ezetimibe treatment. However, 15 days later ALT levels had increased further (ALT $111 \mathrm{U} / \mathrm{L})$, while other laboratory values were unchanged.

His physical examination was negative (for adenopathy, splenomegaly and hepatomegaly, there was only a non specific weakness reported from child). At the end of July, blood tests were repeated and an improvement was noted in all levels, namely AST 46 U/L, ALT 61 U/L, GGT 17 U/L and CK $193 \mathrm{U} / \mathrm{L}$.

In the meantime we had measured hepatitis markers (A, $\mathrm{B}, \mathrm{C}$ ) and also antibodies to cytomegalovirus and to the EBV. The last proved positive for specific antibodies against viral capsid antigens (VCA) IgM and IgG. This result suggested an acute EBV infection.

We and the family decided to test ezetimibe $10 \mathrm{mg} /$ day again. After one month, AST and ALT levels were in a normal range $(<45$ and $<40 \mathrm{U} / \mathrm{L})$. His LDL-C levels were down to $150.8 \mathrm{mg} / \mathrm{dL}$. In order to assess potential contributors to ezetimibe toxicity, this agent not being handled by the cytochrome P-450 system, the activity of conjugating enzymes was assessed. While the child had a normal conjugated bilirubin level $(0.9 \mathrm{mg} / \mathrm{dL})$, his $24 \mathrm{~h}$ urinary D-glucaric acid excretion was $14.1 \mu \mathrm{mol} / \mathrm{g}$ of creatinine (normal range for males, 3.6 - $24.5 \mu \mathrm{mol} / \mathrm{g}$ of creatinine).

\section{Discussion}

This is, to the best of our knowledge, the first report of a child with significant liver side effects from ezetimibe treatment. Unfortunately the morphology and general characteristic of the liver lesion (if visible) could not be evaluated. On the other hand, since ezetimibe does not undergo microsomal P450 metabolism, we evaluated whether conjugative phenomena were normal in this child and we found that he had a normal bilirubin conjugation and a normal excretion of D-glucaric acid [11]. This latter is generally a highly reliable marker of liver conjugating capacity.

Castellone et al [12] reported the case of a woman who developed serious liver damage after 4 months of therapy with ezetimibe $10 \mathrm{mg} /$ day. Since the enzyme pattern was typical of a hepatocyte lesion, it can be excluded, as suggested by Stolk et al [10] that the findings in our child could be due to biliary dysfunction or to an autoimmune phenomenon. Similarly Liu et al [13] reported the case of a 75-yearold Caucasian woman, with a past history of long-standing polycystic kidney disease and an unhealthy life-style (smoking and alcohol) treated for 6 months with ezetimibe $10 \mathrm{mg} /$ day, who also presented elevated levels of liver enzymes.

A further laboratory work, investigating concomitant diseases, possibly of a viral nature, indicated that a recent EBV infection had occurred. This had no clinical manifestations, namely, no parotitis, spleno or hepatomegaly or any evident lymph node enlargement. It should be noted that EBV infections in children, besides the possible association with mononucleosis, may lead to severe hepatitis, certainly not the case in this child. In the majority of young adults, laboratory work generally indicates mild to moderate liver dysfunction, with modestly increased AST, GGT, alkaline phosphatase and bilirubin levels in over $90 \%$ of cases. Interestingly, most studies show normal ALT levels [14, 15]. The acute EBV infection generally resolves in about one month with spontaneous normalization of liver enzymes.

A possible hypothesis is, thus, that ezetimibe may have induced some liver change, ie elevated ALT, and this may have been worsened by the concomitant presence of the EBV infection. In this child, in fact, there was a modest increase of AST, whereas GGT and bilirubin were all in the normal range. The increase of ALT, in this case, may be specifically linked to ezetimibe treatment.

In conclusion, this first report of a clinical case of liver damage in a child, although transient (but liver enzymes remained elevated for over one month after stopping treatment) gives a word of caution in the use of ezetimibe in the younger patient group. The enzyme elevation in the child does not appear to be related to an abnormal handling of the drug by a conjugative mechanism and would thus appear as an idiosyncratic phenomenon in a child with previous EBV virus, up to now not described with ezetimibe, but possibly occurring in rare instances. The observation of a mononucleosis in a child, or for that matter the detection of an EBV infection, should be thus rated as a contraindication to the start of ezetimibe treatment in children, also in view of the considerable worry for parents and doctors, raised by the observation of elevated liver enzymes in growing children.

\section{Conflict of Interest}

None.

\section{References}

1. Rodenburg J, Vissers MN, Wiegman A, van Trotsen- 
burg AS, van der Graaf A, de Groot E, Wijburg FA, et al. Statin treatment in children with familial hypercholesterolemia: the younger, the better. Circulation. 2007;116(6):664-668.

2. National Cholesterol Education Program (NCEP): highlights of the report of the Expert Panel on Blood Cholesterol Levels in Children and Adolescents. Pediatrics. 1992;89(3):495-501.

3. Harper CR, Jacobson TA. The broad spectrum of statin myopathy: from myalgia to rhabdomyolysis. Curr Opin Lipidol. 2007;18(4):401-408.

4. Henck JW, Craft WR, Black A, Colgin J, Anderson JA. Pre- and postnatal toxicity of the HMG-CoA reductase inhibitor atorvastatin in rats. Toxicol Sci. 1998;41(1):8899.

5. Altmann SW, Davis HR, Jr., Zhu LJ, Yao X, Hoos LM, Tetzloff G, Iyer SP, et al. Niemann-Pick C1 Like 1 protein is critical for intestinal cholesterol absorption. Science. 2004;303(5661):1201-1204.

6. Sudhop T, Lutjohann D, von Bergmann K. Sterol transporters: targets of natural sterols and new lipid lowering drugs. Pharmacol Ther. 2005;105(3):333-341.

7. Yeste D, Chacon P, Clemente M, Albisu MA, Gussinye M, Carrascosa A. Ezetimibe as monotherapy in the treatment of hypercholesterolemia in children and adolescents. J Pediatr Endocrinol Metab. 2009;22(6):487-492.
8. Clauss S, Wai KM, Kavey RE, Kuehl K. Ezetimibe treatment of pediatric patients with hypercholesterolemia. J Pediatr. 2009;154(6):869-872.

9. Kosoglou T, Statkevich P, Johnson-Levonas AO, Paolini JF, Bergman AJ, Alton KB. Ezetimibe: a review of its metabolism, pharmacokinetics and drug interactions. Clin Pharmacokinet. 2005;44(5):467-494.

10. Stolk MF, Becx MC, Kuypers KC, Seldenrijk CA. Severe hepatic side effects of ezetimibe. Clin Gastroenterol Hepatol. 2006;4(7):908-911.

11. Mocarelli P, Brambilla P, Colombo L, Marocchi A, Crespi C, Tramacere P, Mondonico A. A new method for Dglucaric acid excretion measurement that is suitable for automated instruments. Clin Chem. 1988;34(11):22832290 .

12. Castellote J, Ariza J, Rota R, Girbau A, Xiol X. Serious drug-induced liver disease secondary to ezetimibe. World J Gastroenterol. 2008;14(32):5098-5099.

13. Liu Q, Tobias H, Petrovic LM. Drug-induced liver injury associated with ezetimibe therapy. Dig Dis Sci. 2007;52(2):602-605.

14. Luzuriaga K, Sullivan JL. Infectious mononucleosis. N Engl J Med. 2010;362(21):1993-2000.

15. Horwitz CA, Burke MD, Grimes P, Tombers J. Hepatic function in mononucleosis induced by Epstein-Barr virus and cytomegalovirus. Clin Chem. 1980;26(2):243-246. 